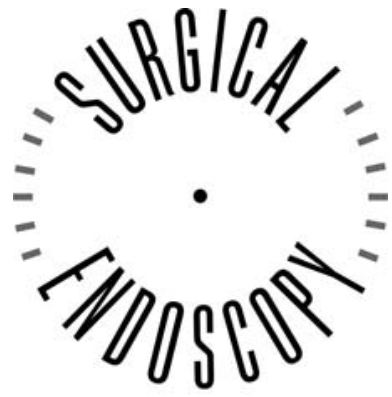

and Other Interventional Techniques

\title{
Analysis of verbal communication during teaching in the operating room and the potentials for surgical training
}

\author{
E. M. Blom, ${ }^{1}$ E. G. G. Verdaasdonk, ${ }^{1,2}$ L. P. S. Stassen, ${ }^{1,2}$ H. G. Stassen, ${ }^{1}$ P. A. Wieringa, ${ }^{1}$ J. Dankelman ${ }^{1}$ \\ ${ }^{1}$ Department of BioMechanical Engineering, Delft University of Technology, Mekelweg 2, 2628, CD, Delft, The Netherlands \\ ${ }^{2}$ Department of Surgery, Reinier de Graaf Group, Delft, The Netherlands
}

Received: 27 September 2006/Accepted: 1 October 2006/Online publication: 7 February 2007

\begin{abstract}
Background: Verbal communication in the operating room during surgical procedures affects team performance, reflects individual skills, and is related to the complexity of the operation process. During the procedural training of surgeons (residents), feedback and guidance is given through verbal communication. A classification method based on structural analysis of the contents was developed to analyze verbal communication. This study aimed to evaluate whether a classification method for the contents of verbal communication in the operating room could provide insight into the teaching processes.

Methods: Eight laparoscopic cholecystectomies were videotaped. Two entire cholecystectomies and the dissection phase of six additional procedures were analyzed by categorization of the communication in terms of type (4 categories: commanding, explaining, questioning, and miscellaneous) and content (9 categories: operation method, location, direction, instrument handling, visualization, anatomy and pathology, general, private, undefinable). The operation was divided into six phases: start, dissection, clipping, separating, control, closing.

Results: Classification of the communication during two entire procedures showed that each phase of the operation was dominated by different kinds of communication. A high percentage of explaining anatomy and pathology was found throughout the whole procedure except for the control and closing phases. In the dissection phases, $60 \%$ of verbal communication concerned explaining. These explaining communication events were divided as follows: $27 \%$ operation method, $19 \%$ anatomy and pathology, 25\% location (positioning of the instrument-tissue interaction), $15 \%$ direction (direction of tissue manipulation), $11 \%$ instrument handling, and 3\% other nonclassified instructions.
\end{abstract}

Correspondence to: E. M. Blom
Conclusion: The proposed classification method is feasible for analyzing verbal communication during surgical procedures. Communication content objectively reflects the interaction between surgeon and resident. This information can potentially be used to specify training needs, and may contribute to the evaluation of different training methods.

Key words: Communication content analysis Observation study - Operating room - Team performance - Training

Verbal communication during surgical procedures affects team performance, reflects individual skills, and is related to the complexity of the operational process [16, 17]. Survey data collected by Helmreich and Schaefer [9] support the idea that interpersonal and communication issues are responsible for many inefficiencies and errors. In aviation, poor interpersonal communication has already been identified as a cause of error [8]. In the operating room, the impact of the communication contents, the way that information is given (verbally or by gestures), and the direction of communication (who communicates with whom) on the surgical process remain unclear. Insight into current communication may improve the overall performance in the operating room, especially the training process. This insight can be used to identify training needs, to develop information supply systems, and to enhance efficiency and safety of surgical team performance. Therefore, a communication classification method is needed.

Analysis of verbal communication is difficult. Communication between operating room personnel is not standardized and thus is susceptible to individual differences. Furthermore, the operating room is a complex environment, and the clinical outcome is influenced by many factors such as equipment, workload, individual (staff) competence, anatomic variations of the patient, 

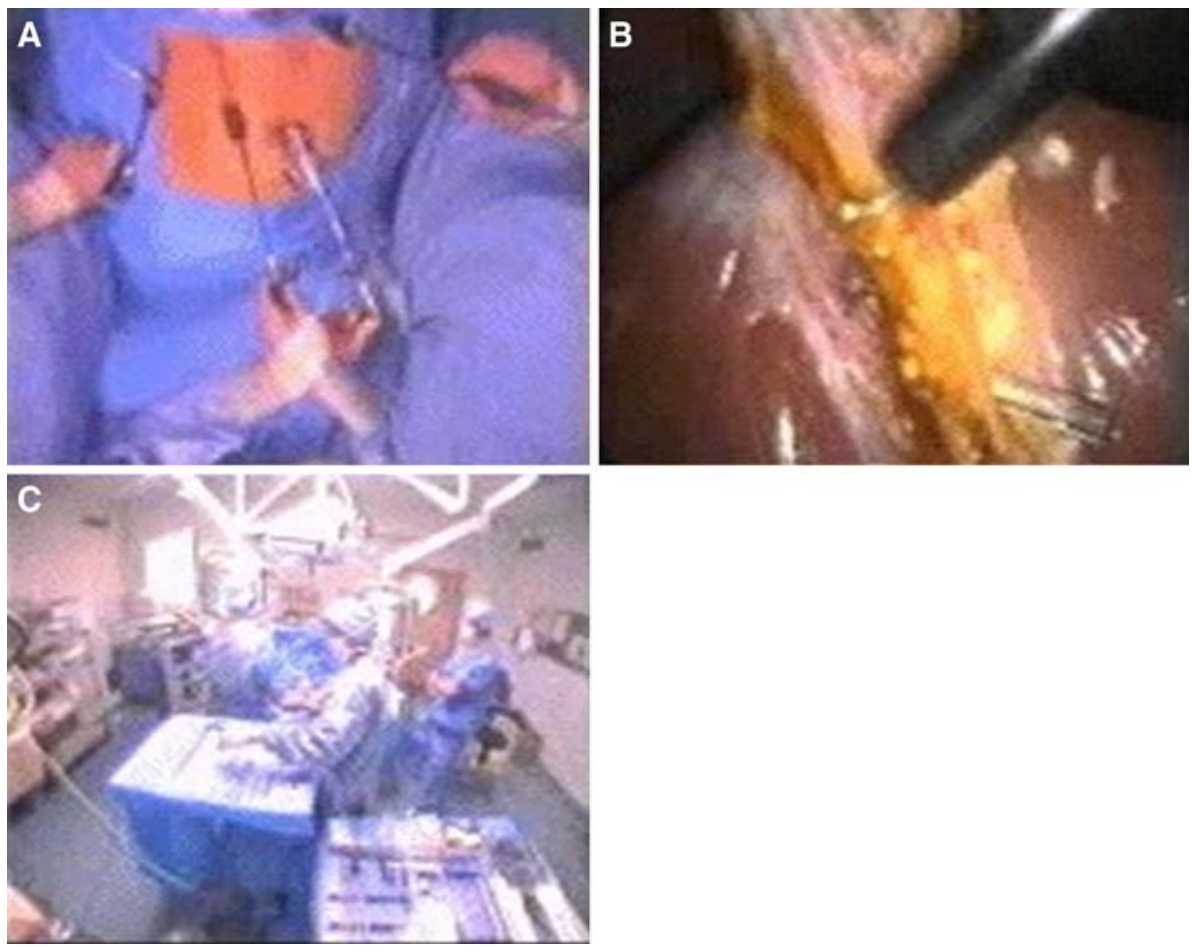

Fig. 1. Video image view of a recorded procedure. a Overview of the surgeon's hands and the external parts of the instruments. b Endoscopic view. c Overall view of the operating room. and the like [16]. Employees with different backgrounds must work together as a team, often under time pressure and sometimes in critical circumstances. The surgeon operates on a patient in cooperation with an assisting surgeon or resident, a scrub nurse, a circulation nurse, and the anesthesiologist. In the educational setting, intensive and often critical information exchange between the different team members in terms of both verbal and nonverbal communication takes place. Gaining insight into current communication in the operating room calls for a standardized communication classification method.

Different methods for analyzing cognitive tasks and communication have been developed [6, 7, 10]. Hauge et al. [7] described a method aimed at registering teaching behavior in the operating room to distinguish between informing, questioning, responding, and setting tone behavior. Lingard et al. [10] described investigations of team communication in the operating room to distinguish patterns that suggest sites of team tension that influence overall team performance. Guerlain et al. [6] developed an extensive software program to analyze audiovisual data from operation procedures. Xiao et al. [17] performed quantitative analysis of team communication based on real-life trauma patient resuscitation. However, none of these reported methods are particularly aimed at analyzing the contents of communication.

To gain insight into the specific contents of verbal communication processes, a new classification method was developed at the Delft University of Technology [2]. The current study aimed to evaluate how analysis of the contents of verbal communication in the operating room may yield insight into teaching processes. Observations were made during laparoscopic cholecystectomies.

\section{Methods}

\section{Video capturing system}

A special video capturing system, developed at the Academic Medical Centre of Amsterdam [15], was used in combination with a headmounted microphone for the surgeon. This video showed three different camera views in the operating room. The first was a detailed view of the surgeon's hands. The second showed the endoscopic image, and the third showed an overall view of the operating room (Fig. 1). The subjects were aware of this monitoring, but did not know that the observation was aimed at verbal communication analysis.

\section{Development of the method}

After thorough observations of several laparoscopic cholecystectomies, communication was classified according to its content on the basis of knowledge gathered during attendance at other procedures. Videotapes of the attended operations also were observed offline. The different categories were discussed with medical specialists and researchers.

In a recent study [2], this new classification method was improved and evaluated (70\% interobserver agreement, $86 \%$ intraobserver agreement). Analysis of communication cannot be accomplished without interpretation, and high interobserver agreement values were difficult to achieve.

\section{Communication classification}

Four communication types were distinguished: explaining, commanding, questioning, and miscellaneous communicating (Table 1). Additionally, the content of the communication type was classified into nine domains: the operation method that should be used (e.g., what procedural steps, which sequence, with how much force), anatomy and pathology, the location of a certain action (description of where to interact with tissue), the direction of the action (in which direction to push or pull tissue), instrument or operating room instrumentation handling, visualization (with the endoscope), general, private, and indefinable. 
Table 1. Different types, contents, and used combinations in the communication classification method

\begin{tabular}{lllll}
\hline $\begin{array}{l}\text { Type } \\
\text { Content }\end{array}$ & Explaining & Questioning & Commanding & \\
\hline Operation method & $\mathrm{X}$ & $\mathrm{X}$ & \\
Anatomy/pathology & $\mathrm{X}$ & $\mathrm{X}$ & & \\
Location & $\mathrm{X}$ & $\mathrm{X}$ & $\mathrm{X}$ & \\
Direction & $\mathrm{X}$ & $\mathrm{X}$ & $\mathrm{X}$ & $\mathrm{X}$ \\
Instrument handling & $\mathrm{X}$ & $\mathrm{X}$ & $\mathrm{X}$ & $\mathrm{X}$ \\
Visualization & $\mathrm{X}$ & $\mathrm{X}$ & $\mathrm{X}$ \\
General & $\mathrm{X}$ & $\mathrm{X}$ & $\mathrm{X}$ \\
Private & & & \\
Undefinable & & & \\
\hline
\end{tabular}

Table 2. Details of the phases of two laparoscopic cholecystectomies

\begin{tabular}{|c|c|c|c|c|c|}
\hline \multirow[b]{2}{*}{ Phase } & \multirow[b]{2}{*}{ Description } & \multicolumn{2}{|c|}{ Total time (min) } & \multicolumn{2}{|c|}{$\begin{array}{l}\text { Communication intensity } \\
\text { (communication events } / \text { min) }\end{array}$} \\
\hline & & Procedure 1 & Procedure 2 & Procedure 1 & Procedure 2 \\
\hline Start & $\begin{array}{l}\text { Starts when the operating team disinfects the } \\
\text { skin and drapes the patient }\end{array}$ & 12 & 10 & 8.2 & 4.8 \\
\hline Dissection & Starts when first instrument is inserted through a trocar & 21 & 11 & 4.8 & 5.1 \\
\hline Clipping & Starts when the first clip is inserted & 14 & 5 & 3.8 & 2.2 \\
\hline Separating & Starts after last clip is placed & 16 & 10 & 5.1 & 3.4 \\
\hline Control & Starts after removal of the gallbladder from the abdomen & 6 & 1 & 5.8 & 9 \\
\hline Closing & $\begin{array}{l}\text { Starts when the surgeon starts to close the wounds } \\
\text { and ends after removal of the drapes }\end{array}$ & & 18 & & 4.4 \\
\hline Total & & 69 & 59 & 5.3 & 4.3 \\
\hline
\end{tabular}

Not all combinations between type and content are possible. For instance a combination of commanding (as type) for anatomy and pathology (as content) does not occur.

In total, communication was divided into 23 categories. All 23 different categories were defined precisely. The categories comprise combinations of type and content domains. For example, "insert the lateral trocar first" is scored as a combination of explaining and operation method, whereas "this gallbladder is inflamed" is scored as explaining and anatomy/pathology. The explaining and location categorization is applied for communication such as "dissect the tissue a little bit more to the left."

In this study, communication aimed at transfer of knowledge from a senior surgeon to a surgical resident is scored as explaining, even if these communication events have the sound of commanding. Commanding about how to adjust the endoscope is scored as commanding visualization in any case.

\section{Scoring system}

Videotapes were digitized and analyzed. Time, phase of the operation, type and contents of communication, and direction (who communicates with whom) were scored with the help of a software program (J-video, University of Maryland, Baltimore, Maryland, USA). The $\mathrm{J}$-video program, a task analysis program, was adjusted to make it applicable for the communication classification method. The 23 different categories and the possibility of scoring other events of importance to the procedure were implemented in the software program. The observer stopped the video immediately after each communication event to have time to make a proper choice between the categories. Every tape was scored twice by the observer. The classification method was applied to both the operating surgeon and the resident under supervision of the surgeon.

\section{Analyzed surgical procedures}

Two entire laparoscopic cholecystectomies and the dissection phase of six additional procedures were videotaped and analyzed. The two entire procedures were performed by a senior surgeon with the assistance of a surgical resident. Each procedure was divided into six phases, as shown in Table 2 and previously described by den Boer et al. [4]. Every phase from the starting phase to the hemorrhage control phase was recorded. Six other procedures were videotaped, but only the dissection phase was analyzed. Focus on this phase seemed to be indicated as the phase in which most manipulations and tissue-instrument interaction take place and the most intensive communication in the training setting is demanded. The dissection phases were performed by six different surgical residents and supervised by a senior, experienced laparoscopic surgeon.

\section{Results}

\section{Results of two entire procedures}

The first analyzed procedure required $69 \mathrm{~min}$. The resident assisting with this procedure had previously assisted in two procedures during his surgical training. In the $69 \mathrm{~min}, 369$ communication events were observed (average of 5.3 communication events per minute). The second procedure consisted of 238 communication events in 56 min (average of 4.3 communication events per minute). The resident assisting with the second procedure had assisted in more than 10 procedures before and had performed two procedures himself guided by another senior surgeon.

Figure 2 shows the scoring frequency per category. For the two procedures, the average number of communication events per minute was 4.5 (range, 2.2-9.0), as shown in Table 2.

The distribution of the communication events for the four types and the nine contents of communication is shown in Table 3 for both procedures in detail. The highest percentage is found for explaining ( $31 \%$ for the first and $37 \%$ for the second procedure). The second 


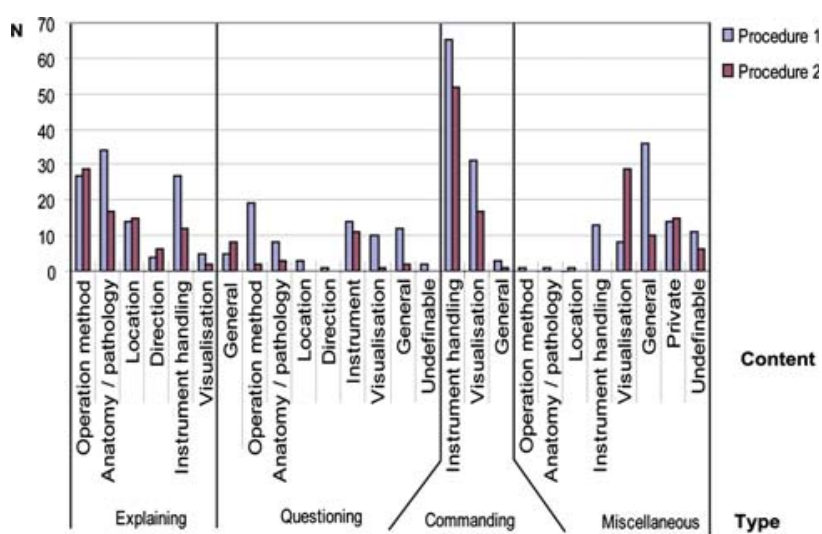

Fig. 2. Total number of communication events per type and content as registered during two laparoscopic cholecystectomies.

Table 3. Types and contents of communication ${ }^{\mathrm{a}}$

\begin{tabular}{llll}
\hline Types & Content & $\begin{array}{l}\text { Procedure } \\
(\%)\end{array}$ & $\begin{array}{l}\text { Procedure 2 } \\
(\%)\end{array}$ \\
\hline Explaining & Total & 31 & 37 \\
& Operation method & 24 & 33 \\
& Anatomy/pathology & 29 & 19 \\
& Location & 12 & 17 \\
& Direction & 4 & 7 \\
& Instrument handling & 23 & 13 \\
& Visualization & 4 & 2 \\
Commanding & General & 4 & 9 \\
Questioning & & 27 & 29 \\
Miscellaneous & & 19 & 8 \\
\end{tabular}

a Type of communication as observed in two entirely monitored laparoscopic cholecystectomies

highest percentage is found for commanding. The lowest percentage is found for questioning. The explaining events of the surgeon in both the procedures were divided into the different content categories shown in Table 3. The highest percentages are found for operating method (24\% and 33\%), anatomy and pathology (29\% and 19\%), and instrument handling (23\% in the first procedure).

For both the procedures, the differences in explaining events during five phases are shown in Fig. 3. For each phase, the division into the contents of explaining communication is visualized. The phase in which the wound is being closed was omitted from consideration of procedure 1 for the practical reason that the sound quality turned out to be insufficient. Explaining of instrument handling is concentrated in the first two phases (start and dissection phases) of the operation. Anatomy was explained most frequently during the dissection and clipping phases, whereas explaining the operation method was scored most often during the last three phases of the operation.

\section{Results of six dissection phases}

The team setting of these procedures varied. Every procedure was performed by a resident guided by one of

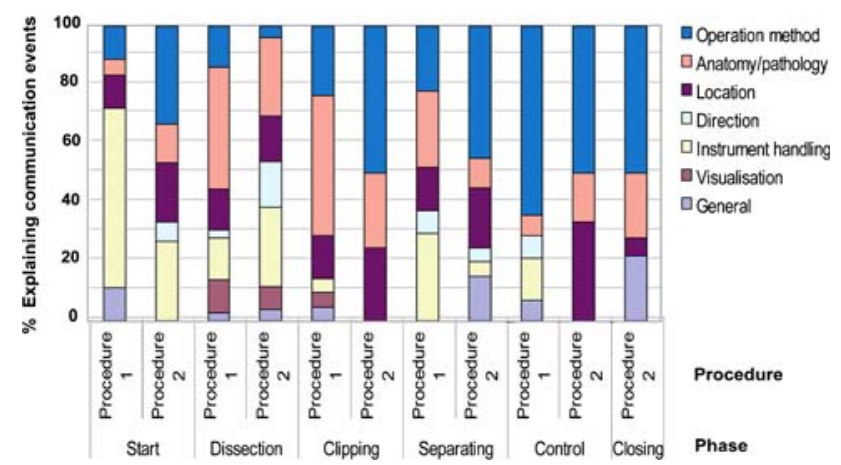

Fig. 3. Distribution of explaining communication events per phase as registered during two laparoscopic cholecystectomies.

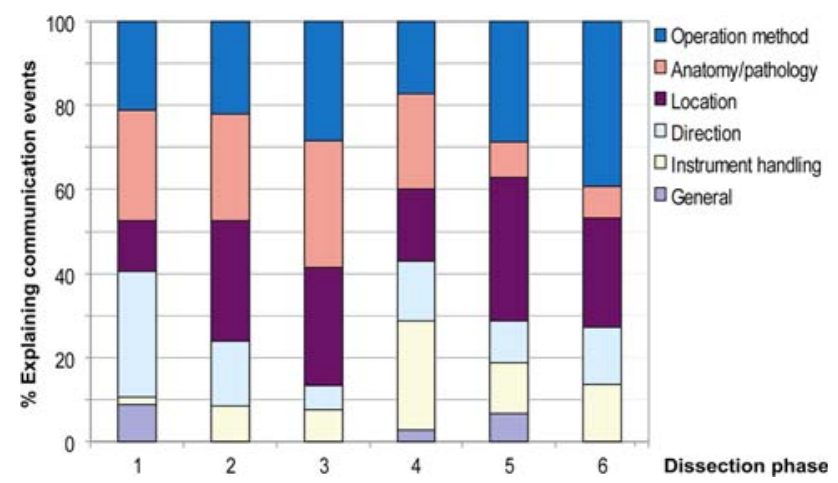

Fig. 4. Distribution of explaining communication events in the dissection phases as registered during six laparoscopic cholecystectomies.

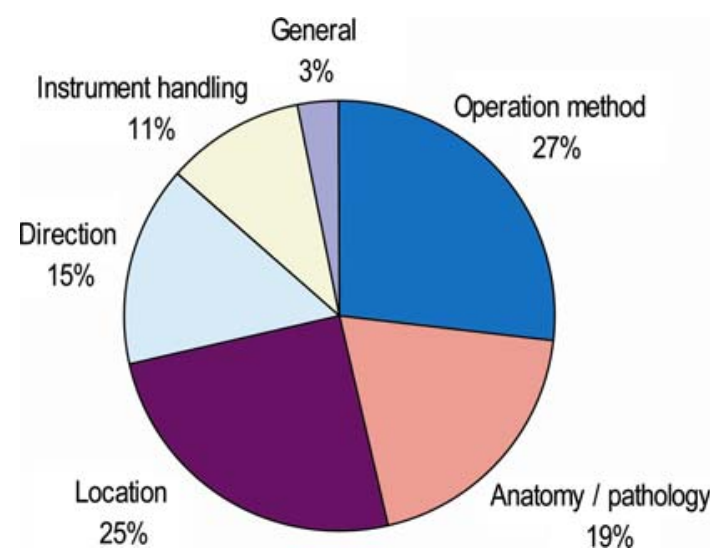

Fig. 5. Average distribution of explaining communication events in the dissection phases as registered during six laparoscopic cholecystectomies.

the four senior surgeons involved in this study. The residents, respectively, had performed $1,1,2,5,6$, and 15 laparoscopic cholecystectomies previously. The scoring of verbal communication aimed at explaining during 20 min of the dissection phase is shown in Figs. 4 and 5. Figure 4 shows the division of the explanation contents per procedure. On the average, $60 \%$ of the communication during the dissection phase of these procedures was related to explaining. All procedures 
show relatively large percentages for explaining of the operation method category. Procedures 1, 2, 3, and 4 also show high percentages for explaining anatomy and pathology, whereas procedures $2,3,5$, and 6 show high percentages for explaining the location.

These explaining communication events were divided as follows: $27 \%$ operation method, $19 \%$ anatomy and pathology, 25\% location, $15 \%$ direction, $11 \%$ instrument handling instructions, and 3\% other nonclassified instructions, as shown in Fig. 5.

\section{Discussion}

This study shows that the described verbal communication classification method is feasible for objective identification of the topics discussed in the operating room. The results show a high percentage of communication related to explaining of the senior surgeon to the surgical resident. Furthermore, explaining communication events were aimed mainly at operating method, anatomy and pathology, and instrument handling. Hence, the training of residents should be focused on these aspects.

\section{Feasibility}

It proved feasible to develop a communication classification method for verbal communication and to apply it for analyzing communication contents in the operating room. The operating room is an environment with complex communication. The proposed classification method facilitates an understanding of these processes.

This approach, however, is time consuming. Gathering of the data required a specially designed video capturing system and the presence of a researcher during the procedure. Nevertheless, with the increasing availability of commercial operating room concepts involving integrated audiovisual communication systems, this process can be fully automated.

Total videotape analyses required approximately three times the time of the original tape. Because the method consists of main types and more detailed contents, the classification should be done in two steps. The first step should recognize the type, and the second step should choose the content. Nevertheless, the interobserver study [2] showed that the classification method was learned quickly by persons with a medical background (surgical residents), and it is the author's opinion that the method also can be learned quickly by researchers without specific medical training.

The sophisticated environment of the operating room makes it difficult to get an overview of the process. The proposed classification method facilitates understanding of the complex communication processes in the operating room. Although nonverbal communication was not included in this method, it reflects most of the teaching behavior. It would be interesting to analyze both the verbal and nonverbal communication optionally in combination with task analysis, debriefing methods, or both. However, analysis of nonverbal communication is even more susceptible to subjectivity and personal interpretation.
In addition to the proposed classification, the different types and contents chosen for a certain evaluation should depend on the goal of the study. When the purpose for using the method is clear, new categories can be added, and unnecessary categories can be omitted. For example, if a researcher is interested only in teaching procedures, only these categories could be scored. It is recommendable to use this method for a larger number of procedures after a more specific research question has been defined, reducing the amount of communication items to be analyzed.

\section{Team communication during training}

Other authors have analyzed communication in the operating room [6, 7]. The importance of good communication has been described by Davies [3]. He stressed its value for safe patient care and team performance. He recommended practicing six components of effective teamwork (situational awareness, problem identification, decision making, workload distribution, time management, and conflict resolution) and self-evaluation of team communication.

Lingard et al. [10] and Minekus et al. [11] reported on analyses of communication failures Lingard et al. [10] observed surgical procedures to distinguish between communication failure events classified as occasion (suboptimal timing), content (insufficiencies or inaccuracies), purpose (lack of resolution), and audience (gaps in group composition). Neither author, however, supplied a communication analysis tool. Our classification method could facilitate evaluations as suggested. Communication analysis also can be combined with task analysis $[4,5]$.

\section{Insight into training needs}

Objective assessment of technical skills in the operating room is an important point of interest in modern surgical teaching. Several methods have been developed for objective assessment of technical skills in the operating room [12]. However, technical skills are only part of a surgeon's competence. Assessment of technical skills needs to be integrated with assessment of cognitive skills and behavioral characteristics such as team performance and decision making. Such evaluation addresses surgical competence as a whole [12].

Aggarwal et al. [1] suggested using a simulated operating room to train both the technical skills and the nontechnical skills such as communication. To assess whether relevant communication is practiced in such a simulated operating room, insight into real communication is imperative. Our classification method could provide a framework for this.

The proposed classification method shows how surgeons teach residents verbally. It shows which topics are being taught during the different phases of the operation. The content of explaining communication indicates the topics that should be practiced before the teaching in the operating room. The high percentages of explaining 
anatomy and pathology (19-34\%) found in the analysis of both entire procedures and the six dissection phases underscore this (Fig. 4). This suggests a specific need for more anatomy and pathology training outside the operating room. A specific curriculum could be developed in which the resident must pass a theoretical test before or after training on a simulator as well as subsequent simulator tests before working in the operating room. Another solution for this specific deficit may be a digital perioperatively available anatomic atlas to enhance and to structure training in the operating room.

More than 25\% of explaining communication events is aimed at explaining the location of instrument-tissue interaction (Fig. 4). The surgeon guides the resident verbally through the procedure by describing exactly where to interact with tissue. Because it is impossible in minimally invasive surgery to point directly to the structures of importance, the surgeon must indicate them by describing color, shape, and the like, which can be complicated and can lead easily to misinterpretation by the receiver. It may be interesting to develop a new "pointing tool." An inexpensive solution may be a sterile laser pointer for pointing to the video monitor. If the surgeon is not scrubbed in, then a "telestrator" can be used on the video monitor.

The development and the effect of using new training tools and methods such as Internet-based education, simulators, and videoconferencing $[1,13]$ could be supported and evaluated with this verbal communication analysis method. The information gained by analyzing verbal communication may be applied in a simulator training curriculum. For instance, when a certain instrument-tissue interaction (e.g., placing a clip on the cystic duct) either does not occur or occurs in the incorrect place (error), the "explanation" used in the operating room can be incorporated into the simulator as proximate (immediate) feedback.

The outcome of our analyses may have been influenced by personal characteristics of the surgical team. Subtle differences were found in the six analyzed dissection phases. These can be explained by differences in the patients, the surgeon's style in guiding the procedure, and the education level of the resident. However, our objective analysis was able to identify types of communication that took place in most procedures irrespective of the differences in the composition of patients and teams.

\section{Objectivity}

Communication cannot be analyzed without interpretation. Hence, our communication analysis contains subjective aspects, although these were largely avoided by using a strict thesaurus, making the method more objective. In a previous study [2], we found an interobserver agreement of 0.7 using this method, which is lower than can be achieved when events or tasks are identified [14] or only types of verbal communication [2, 7]. The agreement in our study is considered satisfactory for giving insight. However, for judgment or for qualifying surgeons/residents, this method must be used with care.
Nevertheless, a wide individual variety exists between surgeons and residents. Sometimes it can be confusing to distinguish between the different types of events. Commanding communication events can be wrapped in questions, and explaining events may have the sound of commanding. For topics such as anatomy, on the one hand, the right classification is obviously explaining. On the other hand, it is almost impossible to distinguish between explaining and commanding for the content "visualization." Therefore, we decided to categorize as commanding all explaining and commanding in combination with the content "visualization."

There is a wide range of application for such a classification method, and it can be specifically beneficial for identifying the different ongoing processes in the operating room. The method for analyzing verbal communication also could be applied for noneducational settings such as team performance assessment, which is an important aspect of improving quality.

\section{Conclusion}

The proposed classification method was developed and used to analyze verbal communication during laparoscopic cholecystectomy. Communication content objectively reflects the interaction between surgeon and resident. Insight into communication contents may be used to specify training needs, and may contribute to the evaluation of different training methods. This study indicates the possibilities for larger research programs aimed at analyzing verbal communication.

Acknowledgments. This research was financially supported by the Reinier the Graaf Group, Delft, The Netherlands, and by TNO Science \& Industry (as part of the OKTO project aimed at the development of innovative information supply tools in the operating room). We thank the Human Factors and Technology research group of University of Maryland for supplying the J-video review and analysis program.

\section{References}

1. Aggarwal R, Undre S, Moorthy K, Vincent C, Darzi A (2004) The simulated operating theatre: comprehensive training for surgical teams. Qual Saf Health Care 13(Suppl 1): i27-i32

2. Blom EM, Verdaasdonk EGG, Stassen LPS, Dankelman J, Remijn SLM, de Graaf MP, Wieringa PA (2005) A new assessment method for verbal communication in the operating room. Proceedings of the 24th EAM [European Annual Manual, Human Decision Making and Manual Control] conference, Athens, Greece, PN05

3. Davies JM (2005) Team communication in the operating room. Acta Anaesthesiol Scand 49: 898-901

4. den Boer KT, Dankelman J, Gouma DJ, Stassen HG (2002) Peroperative analysis of the surgical procedure. Surg Endosc 16: 492-499

5. den Boer KT, de Wit LT, Davids PH, Dankelman J, Gouma DJ (2001) Analysis of the quality and efficiency in learning laparoscopic skills. Surg Endosc 15: 497-503

6. Guerlain S, Adams RB, Turrentine FB, Shin T, Guo H, Collins SR, Calland JF (2005) Assessing team performance in the operating room: development and use of a "black-box" recorder and other tools for the intraoperative environment. J Am Coll Surg 200: 29-37 
7. Hauge LS, Wanzek JA, Godellas C (2001) The reliability of an instrument for identifying and quantifying surgeons' teaching in the operating room. Am J Surg 181: 333-337

8. Helmreich RL (2000) On error management: lessons from aviation. BMJ 320: 781-785

9. Helmreich RL, Schaefer HG (1994) Team performance in the operating room. In: Bogner MS (Human error in Medicine Erlbaum, Hillside, NJ, pp 225-253

10. Lingard L, Reznick R, Espin S, Regehr G, DeVito I (2002) Team communications in the operating room: talk patterns, sites of tension, and implications for novices. Acad Med 77: 232-237

11. Minekus JP, Rozing PM, Nelissen R, Dankelman J (2005) Identifying error pathways during elbow and knee replacements. Clin Orthop Relat Res 437: 121-127

12. Moorthy K, Munz Y, Sarker SK, Darzi A (2003) Objective assessment of technical skills in surgery. BMJ 327: 1032-1037
13. Mutter D, Bouras G, Marescaux J (2005) Digital technologies and quality improvement in cancer surgery. Eur J Surg Oncol 31: 689694

14. Seymour NE, Gallagher AG, Roman SA, O’Brien MK, Bansal VK, Andersen DK, Satava RM (2002) Virtual reality improves operating room performance. Ann Surg 236: 458-464

15. Sjoerdsma W, Meijer DW, Jansen A, den Boer KT, Grimbergen CA (2000) Comparison of efficiencies of three techniques for colon surgery. J Laparoendosc Adv Surg Tech A 10: 47-53

16. Vincent C, Moorthy K, Sarker SK, Chang A, Darzi AW (2004) Systems approaches to surgical quality and safety: from concept to measurement. Ann Surg 239: 475-482

17. Xiao Y, Seagull FJ, Mackenzie C, Ziegert J, Klein KJ (2003) Team communication patterns as measures of team processes: exploring the effects of task urgency and shared team experience. Proceedings of the Human Factors and Ergonomics Society, 47th annual meeting, Denver, CO, USA, pp 1502-1506, 13-17 October 